\title{
Folic acid conjugated cross-linked acrylic polymer (FA-CLAP) hydrogel for site specific delivery of hydrophobic drugs to cancer cells
}

Jisha Jayadevan Pillai ${ }^{1}$, Arun Kumar Theralikattu Thulasidasan², Ruby John Anto ${ }^{2}$, Devika Nandan Chithralekha', Ashwanikumar Narayanan ${ }^{1}$ and Gopalakrishnapillai Sankaramangalam Vinod Kumar ${ }^{{ }^{*}}$

\begin{abstract}
Background: The hydrogel based system is found to be rarely reported for the delivery of hydrophobic drug due to the incompatibility of hydrophilicity of the polymer network and the hydrophobicity of drug. This problem can be solved by preparing semi-interpenetrating network of cross-linked polymer for tuning the hydrophilicity so as to entrap the hydrophobic drugs. The current study is to develop a folic acid conjugated cross-linked pH sensitive, biocompatible polymeric hydrogel to achieve a site specific drug delivery. For that, we have synthesized a folic acid conjugated PEG cross-linked acrylic polymer (FA-CLAP) hydrogel and investigated its loading and release of curcumin. The formed polymer hydrogel was then conjugated with folic acid for the site specific delivery of curcumin to cancer cells and then further characterized and conducted the cell uptake and cytotoxicity studies on human cervical cancer cell lines (HeLa).

Results: In this study, we synthesized folic acid conjugated cross-linked acrylic hydrogel for the delivery of hydrophobic drugs to the cancer site. Poly (ethyleneglycol) (PEG) diacrylate cross-linked acrylic polymer (PAA) was prepared via inverse emulsion polymerization technique and later conjugated it with folic acid (FA-CLAP). Hydrophobic drug curcumin is entrapped into it and investigated the entrapment efficiency. Characterization of synthesized hydogel was done by using Fourier Transform-Infrared spectroscopy (FT-IR), Transmission Electron Microscopy (TEM), Differential Scanning Calorimetry (DSC). Polymerization and folate conjugation was confirmed by FT-IR spectroscopy. The release kinetics of drug from the entrapped form was studied which showed initial burst release followed by sustained release due to swelling and increased cross-linking. In vitro cytotoxicity and cell uptake studies were conducted in human cervical cancer (HeLa) cell lines.
\end{abstract}

Conclusions: Results showed that curcumin entrapped folate conjugated cross-linked acrylic polymer (FA-CLAP) hydrogel showed higher cellular uptake than the non folate conjugated form. So this can be suggested as a better delivery system for site specific release of hydrophobic cancer drugs.

\section{Background}

Hydrogels are polymeric networks having three-dimensional configuration capable of imbibing high amounts of water or biological fluids. Their water absorbing property is mainly attributed to the presence of hydrophilic groups such as $\mathrm{OH},-\mathrm{CONH}-,-\mathrm{CONH}_{2}-$, and $-\mathrm{SO}_{3} \mathrm{H}$ in the polymers. Due to the contribution of these groups and domains in the

\footnotetext{
* Correspondence: gsvinod@rgcb.res.in

'Chemical Biology, Rajiv Gandhi Centre for Biotechnology,

Thiruvananthapuram-695 014, Poojappura, Kerala, India

Full list of author information is available at the end of the article
}

network, the polymer is thus hydrated to different degrees, depending on the aqueous environment and polymer composition [1]. These ionizable functional groups present in it affect its permeability mechanical stability and biocompatibility to a greater extends [2]. Along with that these structures have some common physical properties resembling that of the living tissues, which is attributed to their high water content, soft and rubbery consistency, and low interfacial tension with water or biological fluids [3-5]. The high water content make it soft and wet just like a biological material mimicking the extracellular matrix similar to macromolecular based compound in the human body [6,7]. 
Hydrogel based drug delivery is a type of controlled delivery system were the gel swell resulting in release of drug from the polymer in a controlled manner. As water penetrate through the polymer chain the glass temperature of the polymer decreases and make the hydrogel rubbery [8]. These hydrogels have highly porous structure which helps incorporation of drug into it. The high water content and high porosity help them easy release of the drug within certain hours to days. The porosity of hydrogel can be tuned to the required size of the drug by the addition of cross linker to it. And thus it help in the controlled release of drug $[9,10]$. The polymer used for the preparation of hydrogel is of natural, synthetic and semi-synthetic origin. Even though natural polymers have good bioactive properties [11] they are found to have low mechanical strength so we make use of synthetic polymers because of their good mechanical strength and well-defined structure which can be modified to improve the biocompatibility and biodegradability $[12,13]$.

Polyacrylic acid based polymers are one of such ideal candidates for the synthesis of hydrogel system for the controlled drug delivery because of the swelling behavior in aqueous environment [14]. It is a type of $\mathrm{pH}$ sensitive polymer $[15,16]$ which shows swelling at higher $\mathrm{pH}$ due to the presence of ionizable carboxyl groups in it and can release the drug at neutral $\mathrm{pH}$. The main drawback associated with PAA based drug delivery system is rapid release of drug from it which can be controlled by crosslinking. The cross-linking helps in slow drug release due to small mesh size which can be advantageous for controlled drug delivery applications. Cross-linking also improves the physical properties of the hydrogel including mechanical strength, degradability and diffusivity of drugs from the system. The solubility of polymer in the aqueous environment can also be prevented using crosslinking $[17,18]$.

Curcumin, a naturally occurring yellow coloured polyphenol obtained from the rhizome of the perennial herb Curcuma longa, is found to have potent anticancer properties [19]. It inhibit proliferation and induces apoptosis in various cancer cell lines isolated from malignancies like leukemia, breast, lung, prostate and colon tumors [20-23]. Studies were also done in various tumerogenetic models [24-29] and some clinical trials were also done in patients which confirmed the potential of curcumin as a tool for cancer therapy. But its clinical application becomes limited due to poor water solubility, minimal systemic bioavailability, degradation in alkaline $\mathrm{pH}$ and photo degradation. So the therapeutic efficacy of curcumin can be increased by incorporating curcumin in a biocompatible polymer which enhances the solubility in aqueous solution and extends the release.

One of the main problems associated with cancer therapy is its unwanted side effect towards normal cells along with the cancer cells. An active targeting strategy can improve the therapeutic efficacy of drugs and reduces the side effects [30-34]. For that we have to modify the polymer nanoparticles with certain ligands that have its specific receptor on cancer cell surface. Folate receptor has been extensively investigated for targeting various tumor cells since it is normally expressed in various types of cancer cells [35-38]. So the cross-linked polymeric hydrogel nanoparticles which were structurally modified with folic acid can help in easy targeting and up taking of drugs by the cancer cells.

The hydrogel based system is found to be rarely reported for the delivery of hydrophobic drug due to the incompatibility of hydrophilicity of the polymer network and the hydrophobicity of drug. This problem can be solved by preparing semi-interpenetrating network of cross-linked polymer for tuning the hydrophilicity so as to entrap the hydrophobic drugs. The current study is to develop a folic acid conjugated cross-linked $\mathrm{pH}$ sensitive biocompatible polymeric hydrogel to achieve a site specific drug delivery. For that, we have synthesized a folic acid conjugated PEG cross-linked acrylic polymer (FACLAP) hydrogel and investigated its loading and release of curcumin. Here we used inverse emulsion polymerization technique proposed by Vanderhoff et al. for the polymerization of acrylic acid where an aqueous solution of hydrophilic monomer acrylic acid is dispersed in a continuous lipophilic phase with the aid of surfactants to promote the formation of water in oil $(\mathrm{W} / \mathrm{O})$ emulsion [39]. The formed polymer hydrogel was then conjugated with folic acid for the site specific delivery of curcumin to cancer cells and then further characterized and conducted the cell uptake and cytotoxicity studies on human cervical cancer cell lines (HeLa).

\section{Results and discussion}

\section{Synthesis and characterization of FA-CLAP hydrogel}

Curcumin loaded folic acid conjugated cross-linked acrylic polymer (FA-CLAP) hydrogel were synthesized successfully using inverse emulsion polymerization technique (Figure 1). Inverse emulsion polymerization is a controllable technique used for the preparation of welldefined nanoparticles. In the present study, we prepared acrylic polymer cross-linked with polyethylene glycol diacrylate by inverse micro emulsion polymerization method which is cross-linked with folic acid through ethylenediamine for targeted delivery of curcumin to cancer cells. Inverse polymerization helps in the easy facilitating of free radical polymerization of acrylic monomer with PEG diacrylate in presence of ammonium persulfate. PEG, a highly biocompatible and hydrophilic polymer with low $\mathrm{Tg}$ and polyacrylic acid in the system imparts $\mathrm{pH}$ sensitivity. The hydrogel that we prepared displays $\mathrm{pH}$ sensitive nature which can be exploited for site specific controlled drug delivery. Folic acid is also 

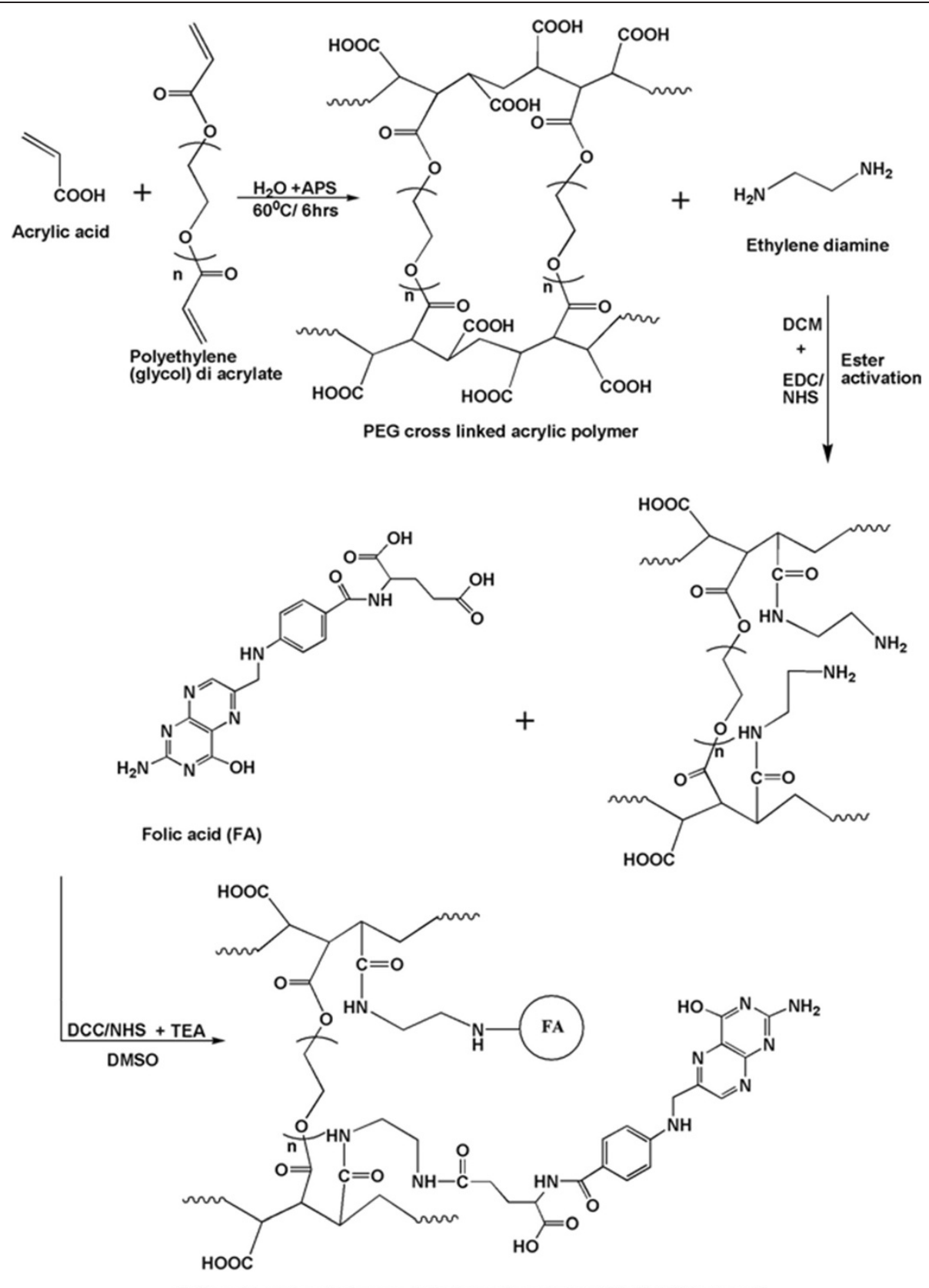

Folic acid conjugated cross linked acrylic polymer (FA-CLAP) hydrogel

Figure 1 Scheme of polymer synthesis. Schematic representation showing the synthesis of folate conjugated cross-linked acrylic polymer hydrogel.

conjugated with the hydrogel that helps in the targeted delivery of the drug encapsulated hydrogel towards the cancer cells since many cancer cells are expressing folic acid on its surface and $\mathrm{pH}$ sensitivity helps in the swelling of the hydrogel in the required site with the sustained release of drug on that particular site. The percentage swelling in a $\mathrm{pH}$ dependant manner was analyzed and given in Additional file 1.

\section{Characterization}

Morphology and the size of the particles were studied using Transmission electron microscopy. The nanoparticles obtained were of size range of 160-190 nm with narrow size distribution (Figure 2). The folic acid conjugated cross-linked polymer hydrogel particle shows a round morphology with nanometric size range. Particle size was found to increase with folic acid conjugation in it. 


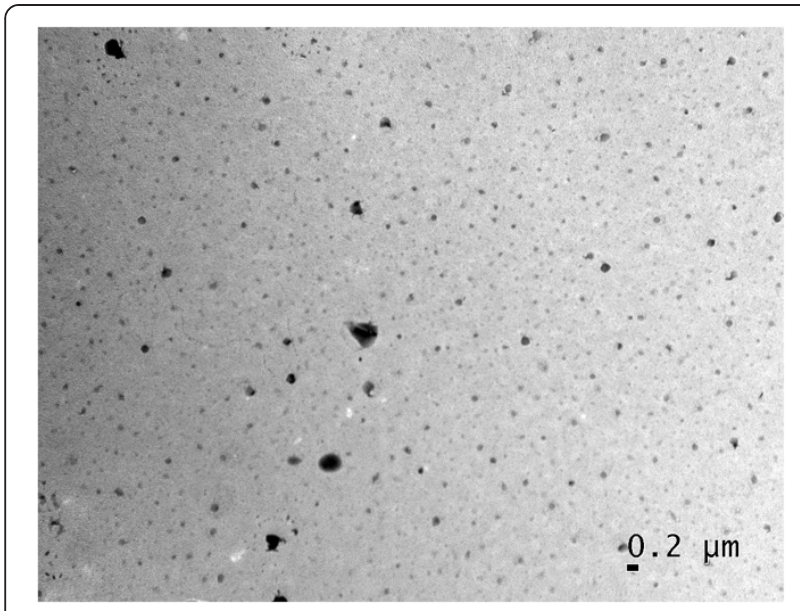

Figure 2 Transmission electron microscope image of (FA-CLAP). TEM image of prepared curcumin loaded FA-CLAP hydrogel nanoparticles.

Role of size in drug delivery is well known. Optimal size should be needed for a drug delivery particle so that it will not easily leak out of the capillaries and also not easily up taken by the macrophages. Here also we developed a particle which is having a size of about $190 \mathrm{~nm}$ that is optimal for the cellular uptake and the folic acid conjugation will help in the active targeted delivery of nanoparticle into the cancer site. Active targeting help in faster accumulation of nanoparticles in the cancer site and help in the release of drug to that particular site [12].

DSC was done to find the thermal behavior of the nanoparticles. DSC of folic acid conjugated cross-liked acrylic polymer and curcumin loaded folic acid conjugated crosslinked polymer were done as showed in Figure 3. Folic acid conjugated polymer shows an endothermic peak at $115^{\circ} \mathrm{C}$ which after loading with curcumin showed a decrease in value (endothermic peak at $85^{\circ} \mathrm{C}$ ) and the nanogel was found to be stable up to $200^{\circ} \mathrm{C}$.

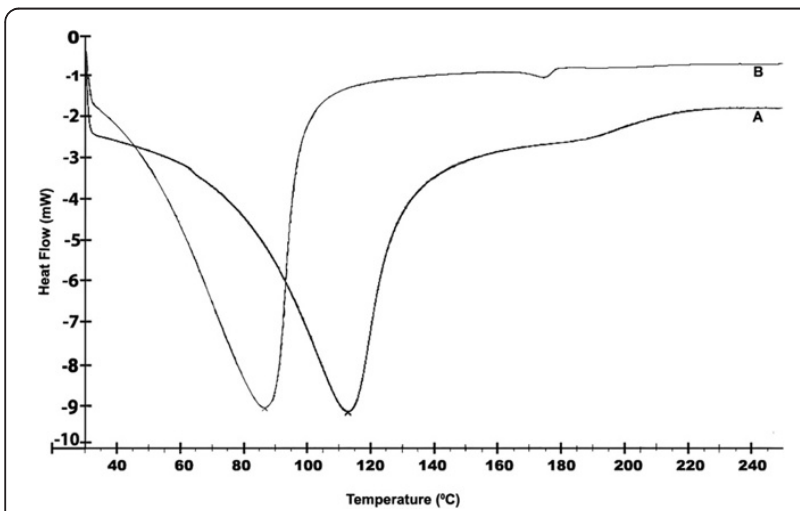

Figure 3 Differential scanning calorimetry (DSC) of hydrogel. DSC of samples (A) FA-CLAP hydrogel (B) Curcumin-entrapped FA-CLAP hydrogel.
The DSC of FA-CLAP hydrogel shows endothermic transition peak at $115^{\circ} \mathrm{C}$ which can be due to the loss of loose and bound water in the hydrogel [40]. The gel appeared to be thermally stable up to $200^{\circ} \mathrm{C}$. Curcumin was loaded to the cross-linked acrylic polymer through physical adsorption by post loading method. And the DSC of curcumin loaded FA-CLAP hydrogel shows an endothermic peak at $85^{\circ} \mathrm{C}$. Cross-linking using PEG diacrylate provides hydrophobicity to the acrylic hydrogel which enhances the uptake of curcumin, a hydrophobic drug. Swelling of the polymer occurs at a $\mathrm{pH}$ above the $\mathrm{pKa}$ of the carboxyl group of acrylic acid. Swelling increases with $\mathrm{COO}$ - groups and decreases with increasing cross-links.

The cross-linking and folate conjugation was confirmed by FTIR spectroscopy. FTIR spectroscopy clearly gives idea about cross-linking and conjugation of folic acid into the polymer (Figure 4). FTIR of cross-linked PAA with EDA (ethylenediamine) was shown in Figure 4(A) in which the peak at $3428.7 \mathrm{~cm}^{-1}$ may be due to N-H stretching of free amino group in ethylenediamine and peak at $1633.6 \mathrm{~cm}^{-1}$ and $1566.7 \mathrm{~cm}^{-1}$ may be of stretching of amide bond I and II that is formed between ethylenediamine and cross-linked PAA which indicates EDA conjugation with the polymer. Peak at $2950.9 \mathrm{~cm}^{-1}$ may be due to aliphatic $\mathrm{C}-\mathrm{H}$ stretching of poly acrylate. FTIR of FA-CLAP hydrogel was shown in Figure 4(B) in which the absorption band at $2929.6 \mathrm{~cm}^{-1}$ is due to asymmetric $\mathrm{C}-\mathrm{H}$ stretching vibration of folic acid. In addition to this other peaks like $1485.3 \mathrm{~cm}^{-1}(-\mathrm{C}=\mathrm{C}$ - aromatic stretching of phenyl ring) and $1411.8 \mathrm{~cm}^{-1}$ (OH deformation of phenyl skeleton) confirm the presence of folic acid in FA-CLAP [41]. Peaks at $1626.5 \mathrm{~cm}^{-1}$ and $1574.9 \mathrm{~cm}^{-1}$ are due to amide I and

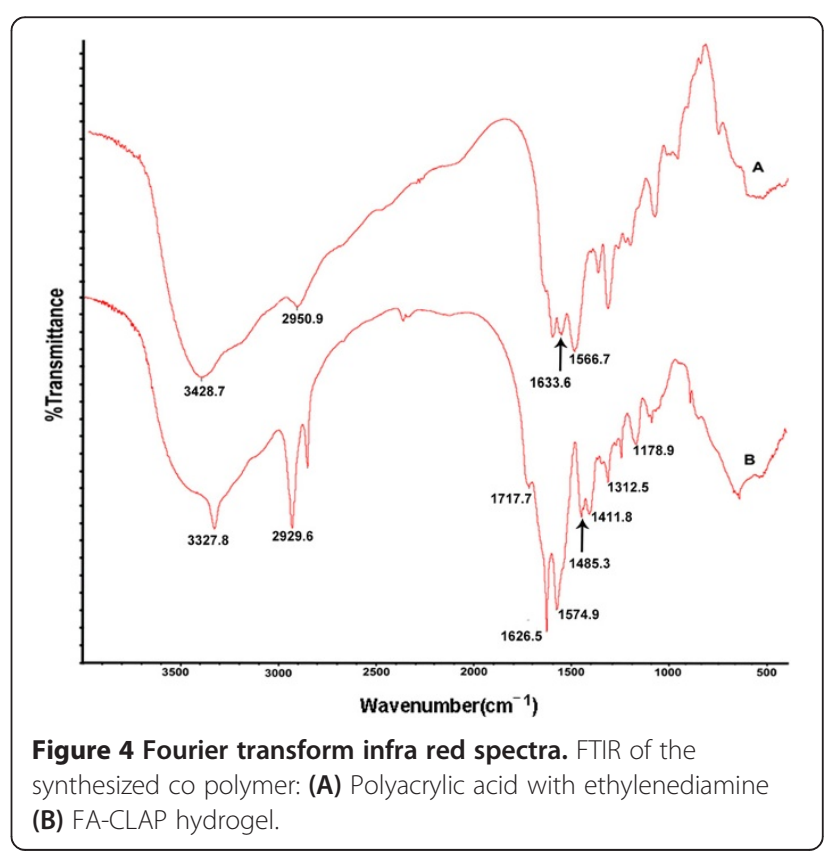


amide II band present in FA-CLAP. Peak at $1312.5 \mathrm{~cm}^{-1}$ may be due to $\mathrm{C}=\mathrm{N}$ medium stretching in the pteridine ring of folic acid which all confirmed the folic acid conjugation with ethylenediamine conjugated cross-linked PAA. It shows peak at $1717.7 \mathrm{~cm}^{-1}$ which may be due to the presence of free $\mathrm{COOH}$ groups in the prepared polymer.

\section{In-vitro drug release}

Curcumin is found to be poorly soluble in water and it form flakes in it. So it is incorporated in a cross-linked polymer hydrogel for delivery to the cancer site. For making targeting more specific, folate conjugation was also done to it. This makes the release of the drug from hydrogel in a controlled manner by the swelling of the gel. Entrapment efficiency of folic acid conjugated cross-linked polymer was found to be $61.2 \pm 1.2 \%$. In vitro release profile of entrapped drug curcumin from FA-CLAP was done and is shown in (Figure 5). Here the polymer showed an initial release of $10 \%$ within 2 hrs and $20-40 \%$ release with in 24 hrs (Higuchi diffusion model [42]) because of swelling of hydrogel and thereafter the release occur in slow sustained manner which might be due to the decrease in the swelling because of increased cross-linking. The release was markedly noticeable from 120-200 hours were $50 \%$ of the increase in drug release was observed. After 200 hours almost $97 \%$ of the drug was released and a steady state was observed. Due to the presence of folic acid, targeted release of drug is possible. Controlled and targeted release of drug from the cross-linked polymer is a desirable property for most of drug delivery applications as it reduces the side effect and increases the bioavailability.

\section{Cell uptake studies}

\section{Curcumin entrapped FA-CLAP show better cellular uptake} compared to free curcumin

We analyzed whether folic acid conjugation to PAA nanocurcumin surface can improve the drug delivery to cancer

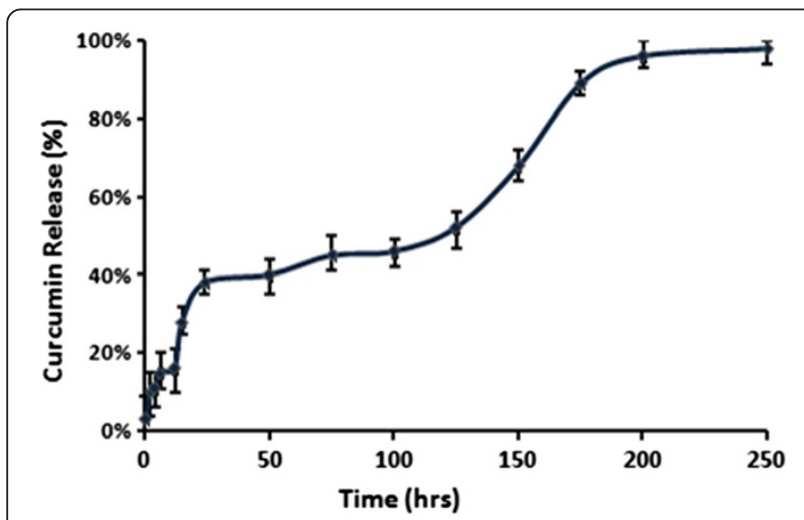

Figure $\mathbf{5}$ In vitro release of curcumin. Release kinetics of curcumin from FA-CLAP hydrogel. cells, since cancer cells over-express folate receptors. The internalization of curcumin to the cells was visualized by confocal microscopy and the results indicate that curcumin entrapped FA-CLAP hydrogel show better cell uptake than free curcumin dissolved in DMSO (Figure 6).

\section{MTT assay \\ Folic acid-conjugated PAA (FA-CLAP) nanocurcumin induce cytotoxicity in HeLa cells}

We compared the efficacy of folic acid-conjugated PAA (FA-CLAP) nanocurcumin with its free counterpart in inducing cell death of HeLa cells. HeLa cells were exposed to free curcumin or folic acid-conjugated PAA nanocurcumin $(5-50 \mu \mathrm{M})$ for $72 \mathrm{~h}$. The nanoformulation imparts high cytotoxicity than free curcumin in dose dependent manner especially for $15 \mu \mathrm{M}, 25 \mu \mathrm{M}$ and $50 \mu \mathrm{M}$. The results indicate that the folic acidconjugated PAA nanocurcumin (FA-CLAP) show comparatively better efficacy in inducing cytotoxicity of HeLa cells than free curcumin dissolved in DMSO (Figure 7).

\section{Acridine orange (AO)/ethidium bromide (EB) staining Conjugation of Folic acid on PAA nanocurcumin slightly enhance the apoptotic effect induced by the latter in HeLa cells}

Ability of curcumin in inducing apoptosis of HeLa cells were assessed by AO/EB staining. HeLa cells were exposed to free curcumin or folic acid-conjugated PAA (FA-CLAP) nanocurcumin $(25 \mu \mathrm{M})$ for $24 \mathrm{~h}$. When treated with $\mathrm{AO} / \mathrm{EB}$ mixture the untreated cells showed bright green chromatin due to AO staining. The damaged membrane of the apoptotic cells allowed EB to get in, giving red color to the nucleus. In Figure 8 we can vividly observe more number of cells in the red colour (6 out of 16 cells, $40 \%$ ) than blank curcumin ( 2 out of 10 cells, $\sim 20 \%)$. The results indicate that conjugation of folic acid on PAA nanocurcumin enhances its apoptotic activity in HeLa cells compared to the free curcumin dissolved in DMSO as assessed by increase in number of EB stained cells (Figure 8).

\section{Conclusions}

Curcumin, which has many medicinal properties mainly, lacks its activity due to its low water solubility. So through this work the delivery of hydrophobic drug curcumin is done by incorporating the drug into crosslinked hydrogel matrix and the cell uptake which was another problem was further enhanced by introducing folic acid into the system. It was found that folic acid conjugated cross-linked hydrogel polymer (FA-CLAP) loaded with curcumin showed better cellular uptake compared to the non-folate hydrogel particles. So this 


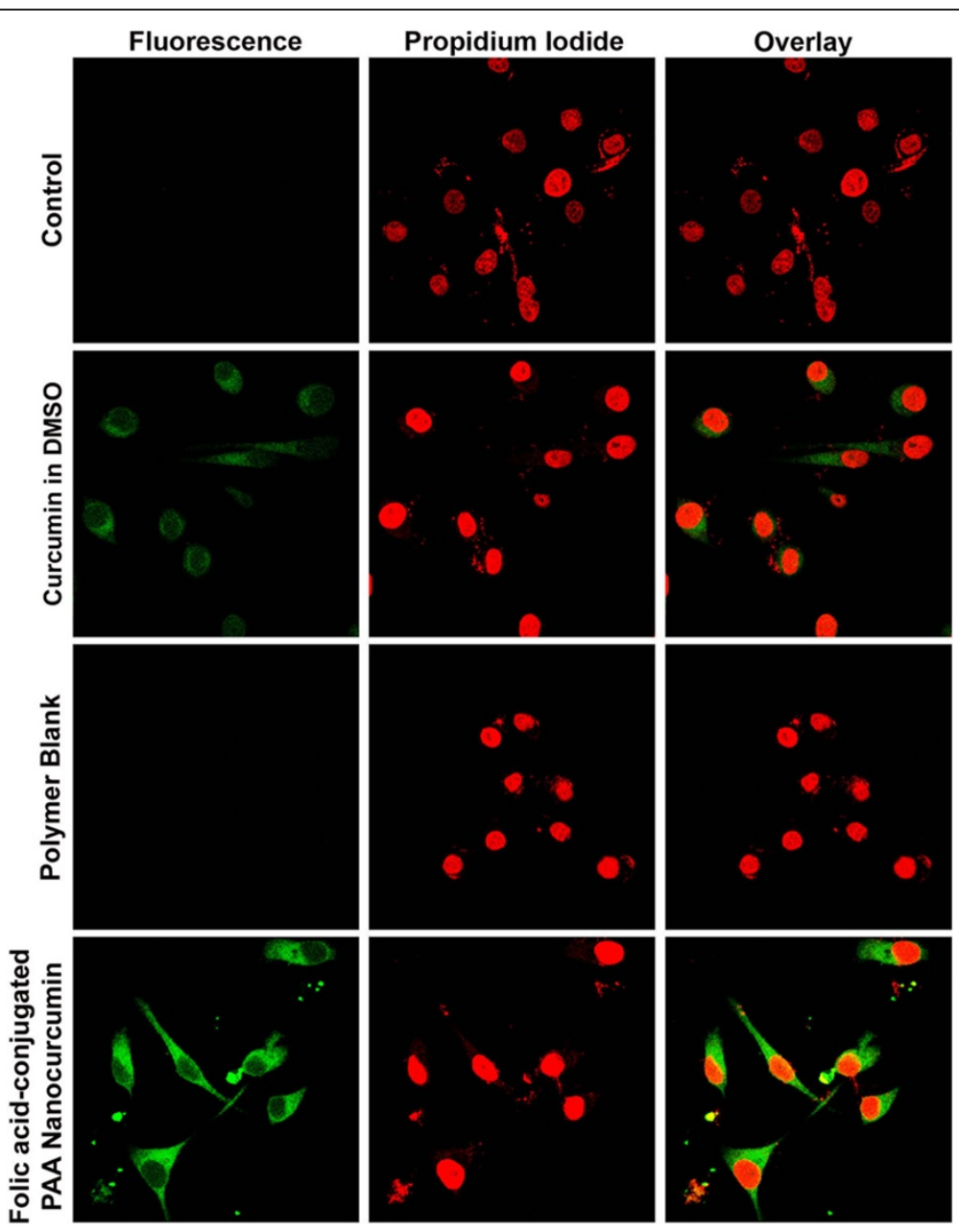

Figure 6 Cellular uptake image. Cell uptake of folic acid-conjugated PAA (FA-CLAP) nanocurcumin and free curcumin: HeLa cells were treated with curcumin formulations as mentioned in materials and methods and confocal images were taken in the FITC channel.

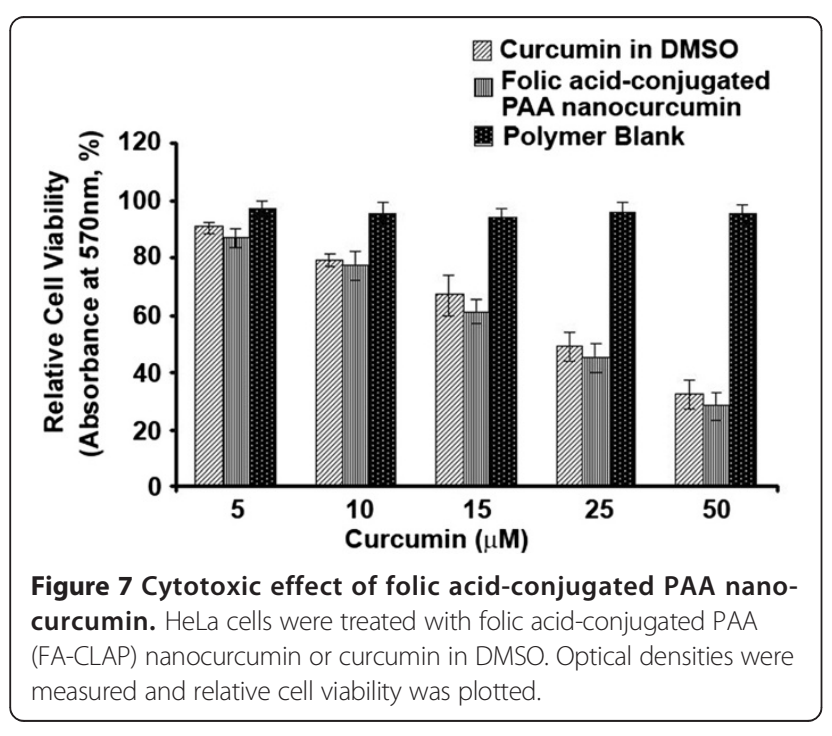

can be used as a better system for the site specific delivery of hydrophobic drugs.

\section{Methods}

\section{Materials}

Acrylic acid ( $\mathrm{Mw} \sim 72)$, cross-linker poly (ethylene glycol) diacrylate $(\mathrm{Mw} \sim 238$,$) , Curcumin, Ammonium$ persulphate (APS), Span 80 (Sorbitan monooleate), Tween 80 (Poly (ethyleneglycol) sorbitan monooleate), 3-(4,5dimethylthiazol-2-yl)-2, 5-diphenyltetrazolium bromide (MTT), ethylenediamine and folic acid were purchased from Sigma-Aldrich, Germany. Acridine orange and ethidium bromide were purchased from Sigma Aldrich. DMEM was purchased from Life Technologies (Grand Island, NY, USA). MTT was purchased from Calbiochem, Germany and Propidium Iodide from Calbiochem, USA. All other reagents and chemicals were of 

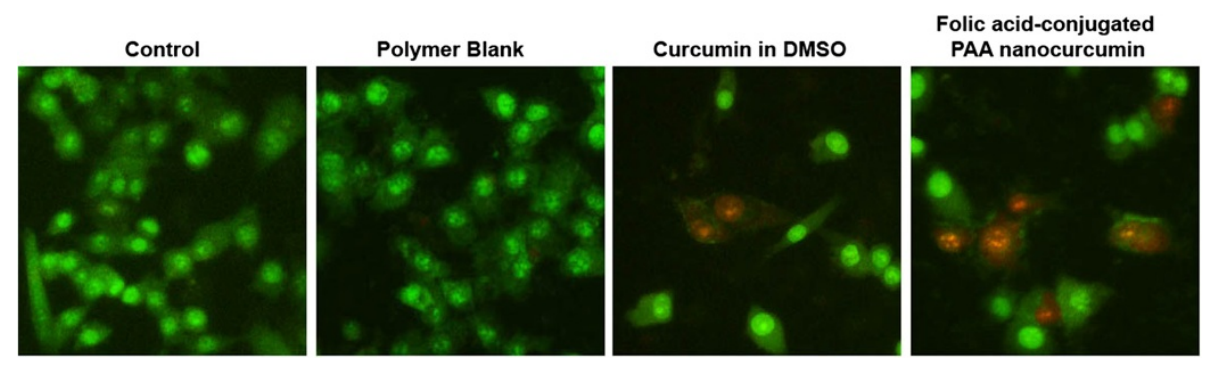

Figure 8 Fluorescence images of HeLa cells treated by free curcumin or folic acid-conjugated PAA (FA-CLAP) nanocurcumin showing apoptosis. HeLa cells were treated with different curcumin formulations for $24 \mathrm{~h}$ and were stained with acridine orange and ethidium bromide solutions as mentioned in materials and methods and fluorescence images were taken using an inverted fluorescent microscope.

analytical grade or above, and used without further purification.

\section{Preparation of folic acid conjugated cross-linked poly- meric nanoparticles}

Preparation of cross-linked acrylic polymer

The cross-linked acrylic polymer (1\%) was prepared by inverse emulsion polymerization technique. Emulsification was done by dispersing aqueous phase consisting of $10 \%$ acrylic acid, $5 \%$ sodium hydroxide and $15 \%$ water with continuous lipophilic phase consisting of liquid paraffin (68\%), emulsifiers (2\%), Span 80 and Tween 80 (75:25 ratio). For cross-linking, $1 \%$ of PEG diacrylate was added to the mixture followed by the addition of initiator, ammonium persulfate (APS). The temperature for polymerization was at $60^{\circ} \mathrm{C}$ for 6 hours. The crosslinked polymeric particles were isolated by centrifugation $(10,000 \mathrm{rpm})$ for $30 \mathrm{~min}$. The isolated polymeric particles were washed several times with hexane and stored for further structural modifications.

\section{Conjugation of folic acid to the prepared cross-linked poly- meric particles (FA-CLAP)}

For the conjugation of folic acid to the prepared crosslinked polymeric particles, first the cross-linked polymeric particle $(850 \mathrm{mg} .1 .2 \mathrm{eq})$ has to be treated with ethylenediamine $(3.2 \mathrm{ml}, 0.65 \mathrm{eq})$ through carbodiimide chemistry for the availability of free amine group for the binding of activated folic acid to it. Simultaneously folic acid (220 mg, $0.5 \mathrm{eq}$ ) has to be ester activated then it is allowed to react with ethylenediamine conjugated crosslinked acrylic hydrogel. A measured amount of this ethylenediamine conjugated cross-linked polymeric particles $(550 \mathrm{mg})$ was dissolved in DMSO. The reaction mixture is kept overnight stirring, for the completion of folic acid conjugation; cross-linked polymeric particles were isolated by centrifugation $(10,000 \mathrm{rpm})$ for $30 \mathrm{~min}$. The isolated folic acid conjugated cross-linked polymeric hydrogel (FA-CLAP) was washed several times and then freeze dried to remove solvent and water. The freeze dried product was stored in vacuum. The polymerization of acrylic acid with PEG diacrylate cross-linking and folic acid conjugation was characterized using DSC and FT-IR spectroscopy. The amount of folic acid conjugated was also estimated (see Additional file 1).

\section{Drug loading}

Loading of curcumin in folic acid conjugated crosslinked polymeric nanoparticles (FA-CLAP) was done by post-polymerization method. $100 \mathrm{mg}$ of the lyophilized powder was dispersed in $10 \mathrm{~mL}$ distilled water. Curcumin was dissolved in chloroform and the drug solution in chloroform was added to the polymeric solution with constant vortexing and sonication. The curcumin loaded FA-CLAP hydrogel was then lyophilized to obtain dried powder.

\section{Characterization of prepared hydrogel nanoparticles}

Morphological analysis of the free and curcumin loaded FA-CLAP nanoparticles were then characterized using transmission electron microscopy (TEM, JEOL 1011, and Japan). The samples of the nanoparticle suspension in water milli- $\mathrm{Q}$ at $25^{\circ} \mathrm{C}$ were dropped on to formvarcoated grids and measurements were taken only after the samples were completely dried.

Folic acid conjugation in the parent polymer was confirmed using FTIR spectroscopy. FTIR spectroscopy was performed on a Spectrum 65 (Perkin Elmer). Spectra were recorded between 4000 and $600 \mathrm{~cm}^{-1}$ wave number range. Dried samples were mixed with $\mathrm{KBr}$ and further compressed in to pellets for making measurements.

Differential Scanning Calorimetry (DSC) was done to analyze the thermal behavior of the FA-CLAP and Curcumin loaded FA-CLAP hydrogel. DSC thermograms obtained were then analyzed using an automatic thermal analyzer system (Pyres 6 DSC, Perkin-Elmer, USA). Samples were placed in standard aluminum pans and heated from 20 to $250^{\circ} \mathrm{C}$ at a rate of $10^{\circ} \mathrm{C} /$ minute under constant purging of $\mathrm{N}_{2}$ at $10 \mathrm{~mL} /$ minute. An empty pan, sealed in the same way as that of the sample, was used as a reference. 


\section{Entrapment efficiency}

A known amount of the curcumin loaded folic acid conjugated cross-linked polymer (FA-CLAP) hydrogel nanoparticles were dissolved in methanol and vigorously vortexed to get a clear solution and it is kept for 24 hours, and then filtered through $0.1 \mu \mathrm{M}$ membrane filter. The absorbance of the filtrate was then taken at $420 \mathrm{~nm}$ by using UV absorbance (Perkin Elmer, USA). The entrapped amount of curcumin was then determined by actual entrapment ratio (AER), expressed in terms of amount of curcumin per weight of nanoparticles [43]. Entrapment efficiency can be calculated by the equation

$$
\text { Entrapment Efficiency }(\%)=\text { AER } / \text { TER } \times 100
$$

$($ AER $=$ Measured drug wt $/$ Nanoparticle wt \& TER $=$ Initial drug wt/ drug wt \& polymer wt). Where (AER) is Actual entrapment ratio and (TER) is Theoretical entrapment ratio. Nanogel wt means the weight of the nanogel with curcumin taken for calculating entrapment efficiency and Initial drug weight means drug initially taken for the entrapment.

\section{In vitro release kinetics}

For in vitro release study, a known amount of curcumin loaded FA-CLAP were dispersed in $10 \mathrm{~mL}$ of P.B.S $(\mathrm{pH} 7.4)$ and was then left in a shaking incubator at 37 $\pm 0.5^{\circ} \mathrm{C}$. A known quantity of sample was then withdrawn and replaced with fresh medium in a predetermined time intervals for maintaining the total volume constant. The amount of curcumin released from the hydrogel nanoparticle was then measured using UV spectrophotometer (Perkin Elmer, USA) at $420 \mathrm{~nm}$.

\section{Cell uptake studies}

Cellular uptake of curcumin and folic acid-conjugated PAA (FA-CLAP) nanocurcumin were studied using confocal microscopy. Briefly, $2.0 \times 10^{4} \mathrm{HeLa}$ cells were grown on cover slips placed in 24 well plates. After overnight incubation, when the cells attained their morphology, they were treated with curcumin dissolved in dimethyl sulfoxide (DMSO) $(25 \mu \mathrm{M})$, folic acid-conjugated PAA nanocurcumin $(25 \mu \mathrm{M})$ was suspended in aqueous medium and blank polymer. After $2 \mathrm{~h}$ of incubation the cells were washed with $1 \mathrm{X}$ PBS, fixed with PFA and the nuclei were stained with propidium iodide and were mounted using DPX. Cells were examined for intracellular fluorescence of curcumin using confocal laser scanning microscope in the FITC channel (488 nm).

\section{MTT assay}

Cytotoxicity studies of free curcumin and folic acidconjugated PAA nanocurcumin were carried out in HeLa cells using MTT assay [44]. HeLa cells were seeded $(3.0 \times$ $10^{3} /$ well) in a 96 -well culture plate and grown for $24 \mathrm{~h}$ before the assay. The cells were then treated with different concentrations of curcumin dissolved in DMSO and folic acid-conjugated PAA nanocurcumin $(5-50 \mu \mathrm{M})$ for $72 \mathrm{~h}$ and then $20 \mu \mathrm{l} \mathrm{MTT}(5 \mathrm{mg} / \mathrm{ml})$ was added in $80 \mu \mathrm{l}$ culture medium to each well. After incubating for $2 \mathrm{~h}$ at $37^{\circ} \mathrm{C}$, cells were lysed using lysis buffer, incubated for $1 \mathrm{~h}$, and the optical densities were measured at $570 \mathrm{~nm}$ using a microplate reader (Bio-Rad Laboratories, Hercules, CA). The relative cell viability in percentage was calculated as:

Relative Cell Viability $=($ A570 of treated samples $/$

A570of untreated samples) $\times 100$

\section{Acridine orange (AO)/ethidium bromide (EB) staining}

Acridine orange/ethidium bromide (AO/EB) double staining was used to detect apoptosis [45]. Briefly, $5 \times 10^{3}$ cells/ well were seeded in a 96-well plate and treated with curcumin in DMSO $(25 \mu \mathrm{M})$ or folic acid-conjugated PAA (FA-CLAP) nanocurcumin $(25 \mu \mathrm{M})$ for $24 \mathrm{~h}$. After washing with $1 \mathrm{X}$ PBS, the cells were stained with acridine orange $(100 \mu \mathrm{g} / \mathrm{ml})$ and ethidium bromide $(100 \mu \mathrm{g} / \mathrm{ml})$ solutions for $2 \mathrm{~min}$. The cells were then washed with $1 \mathrm{X}$ PBS, viewed under an inverted fluorescent microscope (Nikon Eclipse, TE-300) and were photographed.

\section{Additional file}

Additional file 1: Supplementary information.

\section{Competing interests}

The authors declare that they have no competing interests.

\section{Authors' contributions}

JPG synthesized, characterized the polymer and nanoparticles. DNC and NA have written the final manuscript. AKTT had done the biological experiments. RJA participated in evaluation of the biological experiments and supplied information for writing the final manuscript. GSVK planned the whole work and corrected the manuscript. All authors read and approved the final manuscript.

\section{Acknowledgements}

Authors are thankful to Department of Biotechnology, Government of India, for financial support and Arun Kumar T Thulasidasan and Ashwanikumar Narayanan for Council of Scientific and Industrial Research, New Delhi, India for providing Senior Research Fellowship.

\section{Author details}

${ }^{1}$ Chemical Biology, Rajiv Gandhi Centre for Biotechnology, Thiruvananthapuram-695 014, Poojappura, Kerala, India. ${ }^{2}$ Division of Cancer Research, Rajiv Gandhi Centre for Biotechnology, Thiruvananthapuram-695 014, Poojappura, Kerala, India.

Received: 19 March 2014 Accepted: 4 July 2014

Published: 15 July 2014

\section{References}

1. Patel HB, Patel HL, Shah ZH, Modasiya MK: Review on hydrogel nanoparticles in drug delivery. Am J Pharm Res 2011, 1:19-38. 
2. Kim IS, Jeong YI, Kim DH, Lee YH, Kim SH: Albumin release from biodegradable hydrogels composed of dextran and poly (ethylene glycol) macromer. Arch Pharm Res 2001, 24:69-73.

3. Hamidi M, Azadi A, Rafiei P: Hydrogel nanoparticles in drug delivery. Adv Drug Deliv Rev 2008, 60:1638-1649.

4. Ratner BD, Hoffman AS: Synthetic Hydrogels for Biomedical Applications. In Hydrogels for Medical and Related Applications. 31st edition. Edited by Andrade JD. Washington DC: ACS Symposium Series, American Chemical Society; 1976:1-36.

5. Blanco MD, García O, Trigo RM, Teijón JM, Katime I: 5-Fluorouracil release from copolymeric hydrogels of itaconic acid monoester: I. Acrylamide-comonomethyl itaconate. Biomaterials 1996, 17:1061-1067.

6. Chen $R$, Chen $Q$, Huo D, Ding $Y, H u$ Y, Jiang X: In situ formation of chitosan-gold hybrid hydrogel and its application for drug delivery. Colloids Surf B: Biointerfaces 2012, 97:132-137.

7. Liu Y, Chan-Park MB: Hydrogel based on interpenetrating polymer networks of dextran and gelatin for vascular tissue engineering. Biomaterials 2009, 30:196-207.

8. Deepa G, Thulasidasan A, Anto RJ, Pillai JJ, Kumar GSV: Cross-linked acrylic hydrogel for the controlled delivery of hydrophobic drugs in cancer therapy. Int J Nanomedicine 2012, 7:4077-4088.

9. Xiao W, Liu W, Sun J, Dan X, Wei D, Fan H: Ultrasonication and Genipin Cross-Linking to Prepare Novel Silk Fibroin-Gelatin Composite Hydrogel. J Bioact Compat Polym 2012, 27:327-341.

10. Seliktar D: Designing Cell-Compatible Hydrogels for Biomedical Applications. Science 2012, 336:1124-1128.

11. Zhang $L$, Jeong $Y$, Zheng $S$, Jang $S$, Suh H, Kang DH, Kim: Biocompatible and $\mathrm{pH}$-sensitive PEG hydrogels with degradable phosphoester and phosphoamide linkers end-capped with amine for controlled drug delivery. Polym Chem 2013, 4:1084-1094.

12. Khare AR, Peppas NA: Swelling/deswelling of anionic copolymer gels. Biomaterials 1995, 16:559-567.

13. Patenaude $M$, Hoare $T$ : Injectable, mixed natural-synthetic polymer hydrogels with modular properties. Biomacromolecules 2012, 13:369-378.

14. Burugapalli K, Bhatia D, Koul V, Choudhary V: Interpenetrating polymer networks based on poly (acrylic acid) and gelatin I: swelling and thermal behavior. J Appl Polym Sci 2001, 82:217-227.

15. Soppimath K, Aminabhavi T, Dave A, Kumbar S, Rudzinski W: Stimulus-responsive "Smart" hydrogels as novel drug delivery systems*. Drug Dev Ind Pharm 2002, 28:957-974.

16. Gao X, He C, Xiao C, Zhuang X, Chen X: Synthesis and characterization of biodegradable $\mathrm{pH}$-sensitive poly (acrylic acid) hydrogels crosslinked by 2-hydroxyethyl methacrylate modified poly (L-glutamic acid). Mater Lett 2012, 77:74-77.

17. Gupta P, Vermani K, Garg S: Hydrogels: from controlled release to pH-responsive drug delivery. Drug Discov Today 2002, 7:569-579.

18. Hennink W, Van Nostrum C: Novel crosslinking methods to design hydrogels. Adv Drug Deliv Rev 2002, 54:13-36.

19. Kuttan R, Bhanumathy P, Nirmala K, George M: Potential anticancer activity of turmeric (Curcuma longa). Cancer Lett 1985, 29:197-202.

20. Shishodia S, Sethi G, Aggarwal BB: Curcumin: getting back to the roots. Ann N Y Acad Sci 2005, 1056:206-217.

21. Strimpakos AS, Sharma RA: Curcumin: preventive and therapeutic properties in laboratory studies and clinical trials. Antioxidants Redox Signaling 2008, 10:511-546.

22. Anand $P$, Sundaram $C$, Jhurani $S$, Kunnumakkara AB, Aggarwal BB: Curcumin and cancer: an "old-age" disease with an "age-old" solution. Cancer Lett 2008, 267:133-164.

23. Mulik RS, Mönkkönen J, Juvonen RO, Mahadik KR, Paradkar AR: ApoE3 mediated polymeric nanoparticles containing curcumin: Apoptosis induced in vitro anticancer activity against neuroblastoma cells. Int J Pharm 2012, 437:29-41.

24. Tuttle S, Hertan L, Daurio N, Porter S, Kaushic C, Li D, Myamoto S, Lin A, O' Malley BW, Koumenis C: The chemopreventive and clinically used agent curcumin sensitizes HPV-but not HPV+ HNSCC to ionizing radiation, in vitro and in a mouse orthotopic model. Canc Biol Ther 2012, 13:0-1.

25. Chuang S, Kuo M, Hsu C, Chen C, Lin J, Lai G, Hsieh C, Cheng A: Curcumin-containing diet inhibits diethylnitrosamine-induced murine hepatocarcinogenesis. Carcinogenesis 2000, 21:331-335.

26. Kawamori T, Lubet R, Steele VE, Kelloff GJ, Kaskey RB, Rao CV, Reddy BS: Chemopreventive effect of curcumin, a naturally occurring anti-inflammatory agent, during the promotion/progression stages of colon cancer. Cancer Res 1999, 59:597.

27. Inano H, Onoda M, Inafuku N, Kubota M, Kamada Y, Osawa T, Kobayashi H, Wakabayashi $\mathrm{K}$ : Chemoprevention by curcumin during the promotion stage of tumorigenesis of mammary gland in rats irradiated with $\gamma$-rays. Carcinogenesis 1999, 20:1011-1018.

28. Singh SV, Hu X, Srivastava SK, Singh M, Xia H, Orchard JL, Zaren HA: Mechanism of inhibition of benzo [a] pyrene-induced forestomach cancer in mice by dietary curcumin. Carcinogenesis 1998, 19:1357-1360.

29. Li N, Chen X, Liao J, Yang G, Wang S, Josephson Y, Han C, Chen J, Huang MT, Yang CS: Inhibition of 7, 12-dimethylbenz [a] anthracene (DMBA)-induced oral carcinogenesis in hamsters by tea and curcumin. Carcinogenesis 2002, 23:1307-1313

30. Nasongkla N, Bey E, Ren J, Ai H, Khemtong C, Guthi JS, Chin SF, Sherry AD, Boothman DA, Gao J: Multifunctional polymeric micelles as cancer-targeted, MRI-ultrasensitive drug delivery systems. Nano Lett 2006, 6:2427-2430.

31. Nair KL, Sankar J, Nair AS, Kumar GSV: Evaluation of triblock copolymeric micelles of $\delta$-valerolactone and poly (ethylene glycol) as a competent vector for doxorubicin delivery against cancer. J Nanobiotechnology 2011, 9:42.

32. Lilach $V$, Itai $B$ : In vivo characteristics of targeted drug-carrying filamentous bacteriophage nanomedicines. J Nanobiotechnology 2011, 9:58.

33. Mehmet HU, Seta K, Bernhard S, Uwe BS: Characterization of CurcuEmulsomes: nanoformulation for enhanced solubility and delivery of curcumin. J Nanobiotechnology 2013, 11:37

34. Deepa G, Ashwanikumar N, Pillai JJ, Kumar GSV: Polymer nanoparticles-a novel strategy for administration of paclitaxel in cancer chemotherapy. Curr Med Chem 2012, 19:6207-6213.

35. Blanco MD, Guerrero S, Benito M, Fernández A, Teijón C, Olmo R, Katime I, Teijón JM: In vitro and in vivo evaluation of a folate-targeted copolymeric submicrohydrogel based on $\mathrm{n}$-isopropylacrylamide as 5-fluorouracil delivery system. Polymers 2011, 3:1107-1125.

36. Roger E, Kalscheuer S, Kirtane A, Guru BR, Grill AE, Whittum-Hudson J, Panyam J: Folic acid-functionalized nanoparticles for enhanced oral drug delivery. Mol Pharm 2012, 9:2103-2110.

37. Kumar M, Singh G, Arora V, Mewar S, Sharma U, Jagannathan N, Sapra S, Dinda A, Kharbanda S, Singh H: Cellular interaction of folic acid conjugated superparamagnetic iron oxide nanoparticles and its use as contrast agent for targeted magnetic imaging of tumor cells. Int $J$ Nanomedicine 2012, 7:3503-3516.

38. Choi SK, Thomas TP, Li MH, Desai A, Kotlyar A, Baker JR: Photochemical release of methotrexate from folate receptor-targeting PAMAM dendrimer nanoconjugate. Photochem Photobio/ Sci 2012, 11:653-660.

39. Vanderhoff J, Bradford E, Tarkowski H, Shaffer J, Wiley R: Inverse emulsion polymerization. Adv Chem 1962, 34:32-51

40. Ravichandran P, Shantha KL, Rao KP: Preparation, swelling characteristics and evaluation of hydrogels for stomach specific drug delivery. Int J Pharm 1997, 154:89-94.

41. Huang H, Yuan Q, Shah JS, Misra RDK: A new family of folate-decorated and carbon nanotube-mediated drug delivery system: synthesis and drug delivery response. Adv Drug Deliv Rev 2011, 63:1332-1339.

42. Glavas-Dodov M, Goracinova K, Mladenovska K, Fredro-Kumbaradzi E: Release profile of lidocaine $\mathrm{HCl}$ from topical liposomal gel formulation. Int J Pharm 2002, 242:381-384

43. Qiu Y, Park K: Environment-sensitive hydrogels for drug delivery. Adv Drug Deliv Rev 2001, 53:321-339.

44. Anto RJ, Venkatraman M, Karunagaran D: Inhibition of NF- B sensitizes A431 cells to epidermal growth factor-induced apoptosis, whereas its activation by ectopic expression of RelA confers resistance. J Biol Chem 2003, 278:25490-25498.

45. Ribble D, Goldstein NB, Norris DA, Shellman YG: A simple technique for quantifying apoptosis in 96-well plates. BMC Biotechnol 2005, 5:12.

doi:10.1186/1477-3155-12-25

Cite this article as: Pillai et al:: Folic acid conjugated cross-linked acrylic polymer (FA-CLAP) hydrogel for site specific delivery of hydrophobic drugs to cancer cells. Journal of Nanobiotechnology 2014 12:25. 